\title{
Una alternativa de auditoría en psiquiatría más cercana a Europa y al residente
}

\author{
Rosa M. Molina-Ruiz, James G. Strachan, Guillermo Lahera-Forteza, Juan José López-Ibor
}

Introducción. En este trabajo se presenta la experiencia de una auditoría externa piloto realizada en el Servicio de Psiquiatría del Hospital Príncipe de Asturias de Alcalá de Henares, y los beneficios derivados del modelo de la UEMS (Unión Europea de Médicos Especialistas) aquí expuesto. En España, los sistemas de evaluación y calidad de formación de los especialistas están bien establecidos. Existen todavía aspectos que se pueden mejorar. El modelo de auditoría de la UEMS reconoce la diversidad de programas de los distintos países miembros, pero trata de estandarizar la calidad de la formación mediante el proceso de auditorías externas.

Objetivo. Mostrar un modelo más centrado en la formación del residente, con mayor participación de éste y de las sociedades profesionales y científicas.

Materiales y métodos. Se expone la experiencia de auditoría externa realizada en una unidad de psiquiatría siguiendo el modelo de la UEMS.

Resultados. Se analizan los beneficios de este tipo de auditorías en el proceso de revisión de la formación de los residentes.

Conclusión. Este tipo de auditorías favorece la supervisión del sistema de formación de residentes y promueve el acercamiento a los estándares de calidad de la Unión Europea, garantizando que los residentes obtengan el mejor nivel de formación.

Palabras clave. Auditoría externa. Evaluación formativa. Formación del residente. Programa MIR. Supervisión. UEMS.

\section{An alternative way of auditing in psychiatry, closer to Europe and the trainee}

Introduction. This paper relates the experience of a pilot external audit visit conducted at the University Hospital Príncipe de Asturias in Alcalá de Henares and its benefits, following the UEMS (Union Européenne des Médecins Spécialistes) model. In Spain, systems of evaluation and quality of the specialists training are well established. Some aspects can be still improved. The UEMS model of audit, recognises the diversity of programs in member countries, but is trying to standardize the quality of training through external audits.

Aim. To show a model that mainly focuses on the training of residents with a greater participation of them and professional and scientific societies.

Materials and methods. To exhibit the experience of an external audit visit in a Psychiatry unit following the UEMS model. Results. Multiple benefits derive from this kind of audits in the revision and improvement of the residents' training.

Conclusion. This kind of audits provides an assessment of the resident's training system and encourages movement towards European Union standards of quality, ensuring that all trainees get the highest standard of training which can be provided.

Key words. Audit visit. MIR program. Psychiatry training. Supervision. Training assessment. UEMS.

\section{Introducción}

Los métodos de enseñanza y aprendizaje de psiquiatría en España han sido renovados en los últimos tiempos y se han introducido planes de auditoría que llevan funcionando desde hace más de treinta años. La especialidad de psiquiatría en España no se estableció hasta 1955, con la Ley de Especialida- des, y el primer programa para la formación de los psiquiatras no se publicó de forma explícita hasta después de la promulgación del real decreto de 1978, con la constitución de las comisiones nacionales de especialidad (CNE). Se elabora así, por primera vez, un programa de formación en el que se fija un periodo de cuatro años y se establece la necesidad de acreditar adecuadamente los centros do-
Royal Edinburgh Hospital; UEMS Board of Psychiatry; Edimburgo, Reino Unido (J.G. Strachan) Servicio de Psiquiatría; Hospital Universitario Príncipe de Asturias; Alcalá de Henares, Madrid (G. Lahera-Forteza). Instituto de Psiquiatría y Salud Mental; Hospital Clínico San Carlos; Madrid (R.M. Molina-Ruiz, J.J. López-lbor) Departamento de Psiquiatría y Psicología Médica; Facultad de Medicina; Universidad Complutense de Madrid (J.J. López-Ibor). Instituto de Investigación Sanitaria; Hospital Clínico San Carlos; Madrid (J.J. López-Ibor). Centro de Investigación en Red de Salud Mental, CIBERSAM (J.J. López-lbor); Madrid, España.

Correspondencia:

Dra. Rosa M. Molina Ruiz.

Servicio de Psiquiatría. Centro de Especialidades. Modesto Lafuente, 21. E-28003 Madrid.

E-mail:

rosamolina18@hotmail.com

Agradecimientos:

Prof. M. Gómez-Beneyto, jefe de servicio del Hospital Clínico Universitario de Valencia y presidente de la Comisión Nacional de Psiquiatría, por su interés y dedicación en los temas de

formación. A. Fernández Liria, jefe de servicio del Hospital Príncipe de Asturias de Madrid, y Prof.

$P$. Hodiamont, vicepresidente del European Board of Psychiatry (UEMS), por su enorme interés y esfuerzo en que toda esta experiencia se llevase a cabo. Dr. R. Campos Ródenas, especialista de área, profesor asociado y director del programa de formación de residentes de psiquiatría del Hospital Clínico de Zaragoza, por su guía y recomendaciones.

Conflicto de intereses: No declarado

Competing interests: None declared.

(C) 2013 FEM 
centes. El Comité Nacional elaboró el primer programa formativo de psiquiatría en 1986 [1]. También se establece que en cada centro con unidades docentes acreditadas debe existir una comisión de docencia, con el objetivo de organizar la formación, supervisar su aplicación práctica y controlar el cumplimiento de los objetivos previstos en los programas formativos [2]. Las unidades docentes (UD) son acreditadas por el Ministerio de Sanidad y Consumo según el procedimiento regulado en el artículo 26.3 de la Ley 44/2003, de 21 de noviembre, con sujeción a los requisitos básicos y específicos de acreditación, aprobados con carácter general por los Ministerios de Sanidad y de Educación [2,3].

La Comisión Nacional (CN) de Psiquiatría es la responsable de la elaboración del programa nacional de la especialidad y los requisitos de acreditación de las UD $[4,6]$. Se compone de nueve profesionales altamente cualificados en estas materias que representan al Ministerio de Educación, al Consejo Nacional de Colegios de Médicos, a las comunidades autónomas y a las asociaciones científicas, así como dos representantes de los residentes (MIR) de psiquiatría. Estos dos últimos representantes, sin embargo, no proceden de una asociación de residentes de psiquiatría, ya que los dos intentos de asociación de ámbito nacional, no han tenido continuidad en nuestro país - tan sólo entre los años 2001 y 2003 estuvo funcionando la AERP (Asociación Española de Residentes de Psiquiatría) - [7]. De esta manera, queda a cargo de la CN la elección de los representantes de los MIR.

En los últimos cinco años, y en el marco de mejora de la calidad del Sistema Nacional de Salud (SNS), se han implementado los planes de auditoría específicos y programas de formación de auditores. Con la participación de sociedades científicas y personas expertas se elaboran informes de estándares y recomendaciones en calidad y seguridad [8,9]. Además, durante el año 2006, se ha realizado una actualización de los manuales de auditorías para adaptarlos a los nuevos programas de las especialidades y los correspondientes requisitos técnicos de las UD [10].

Para que una red de servicios psiquiátricos (circuito asistencial) sea acreditada como UD, necesita cumplir varios requisitos: recursos asistenciales, de la organización de la unidad, de la actividad asistencial, docente y de investigación, del seguimiento de la guía de formación de especialistas, del control del cumplimiento del programa de formación, y de la encuesta de satisfacción o entrevista [11].

En lo referente al seguimiento, evaluación y calificación del residente (evaluación de la formación), futuro especialista en psiquiatría, se lleva a cabo mediante un proceso de evaluación continuada/formativa, que permite constatar el progreso en el aprendizaje, competencia, etc. El tutor, como responsable, cumplimentará informes normalizados. Se revisarán las rotaciones realizadas, la docencia, la investigación, las guardias, etc. La evaluación anual se lleva a cabo por el comité de evaluación y sus resultados se trasladan a la comisión de docencia para que proceda a su publicación. Dicho comité está formado por el jefe de estudios, el presidente de la subcomisión, el tutor del residente, un profesional que preste servicios en el centro con el título de especialista que corresponda, y uno de los vocales de la comisión de docencia designado por la comunidad autónoma [1].

Con respecto a otros países de la Unión Europea encontramos que éstos poseen procesos de auditoría muy diversos, pero desde la creación de la UEMS (Unión Europea de Médicos Especialistas) en 1958, se está poniendo en marcha un proceso de auditoría que difiere de los habituales y se centra especialmente en la formación específica del residente [12]. La UEMS representa a las asociaciones médicas de Europa, así como a sus países asociados. Su principal objetivo consiste en mejorar la calidad de la atención médica especializada en Europa, armonizando y mejorando la formación posgraduada y la formación médica continuada (FMC). Desde la creación de la sección de psiquiatría, establecida en 1992, la UEMS ha desarrollado varios documentos en relación a los estándares y la evaluación de la especialidad. De gran interés es el $E u$ ropean Framework for Competencies in Psychiatry (marco europeo de competencias en psiquiatría) y el Charter on training of medical specialists in the $E U$ (estatutos de la formación del médico especialista), que desarrollan los objetivos formativos [13, 14]. Asimismo ha realizado grandes esfuerzos para fomentar la auditoría, poniendo especial énfasis en los siguientes aspectos: dispositivos de formación, reconocimiento de la necesidad de tiempo y asignación de recursos. Igualmente ha desarrollado una serie de criterios para el control de calidad: ausencia de intereses comerciales, verificación de la asistencia, evaluación de los asistentes, evaluación de los proveedores y realización de una memoria [15]. Como aspecto más destacable, la UEMS otorga un papel fundamental a las asociaciones profesionales y científicas, así como a los residentes, evitando que las autoridades administrativas tengan más que decir acerca de la formación de los especialistas que ellos mismos. De hecho, la UEMS mantiene una estrecha relación con la EFPT (Federación Europea de Residentes de Psiquiatría), fundada en 1992 para 
dar respuesta a la necesidad de representantes de residentes de psiquiatría en la UEMS. La labor de la EFPT se centra en la formación de los residentes, dándoles una voz relevante y permitiendo su participación en todo el proceso de su formación [16].

La UEMS ha adoptado este sistema como modelo de buena práctica para todos los centros docentes y trata de extender y animar a otros países a seguir este procedimiento de auditorias externas, como forma de comprobar la adhesión al programa nacional o internacional vigente. De hecho, una primera experiencia se realizó en Alemania en el año 2005 [17], y en 2011 se ha repetido en España por iniciativa de nuestros representantes en la UEMS -Sociedad Española de Psiquiatría (SEP) y Asociación Española de Neuropsiquiatría (AEN)-, quienes invitaron a la UEMS a realizarlo en el Hospital Universitario Príncipe de Asturias (HUPA) de Madrid.

Esta primera experiencia de auditoría externa en nuestra especialidad ha servido para mostrar el proceso de evaluación seguido por otros países de la UE, con el fin de incorporar a nuestro modelo aquellos aspectos más relevantes.

\section{Materiales y métodos}

\section{Proceso general para la realización en España de la auditoria externa de psiquiatría}

La auditoria externa realizada en el HUPA se realizó con la participación de los coordinadores de dicho hospital, tres miembros de cada una de las asociaciones representadas en la UEMS (SEP y AEN) y un comité evaluador formado por dos representantes de la UEMS y un residente de psiquiatría ajeno al hospital (generalmente en su último año de residencia). Este último debería ser elegido dentro de la asociación nacional de residentes, para participar como parte del comité evaluador, durante la auditoría y como parte central en el mismo. En España no existe actualmente tal asociación nacional de residentes, por lo que la persona elegida fue a cargo de la CN y ratificado por la SEP y la AEN.

Para clarificar las características del centro, el alcance y los límites de la intervención que podía ofrecerse, el primer paso fue realizar un esquema de la evaluación de la formación (Training Scheme Assessment Form) [18]. Éste incluye información relativa a programas de formación, organización de las distintas unidades, número de residentes, número de psiquiatras senior y supervisores/tutores, oportunidades de investigación para los residentes, supervisión en los distintos campos, ambiente du- rante la formación, facilidades de formación, evaluación de la residencia, etc., similar en la mayoría de los aspectos cubiertos al esquema de auditoría docente de psiquiatría [11].

En una segunda fase, tras la cumplimentación y recepción del esquema de evaluación por el comité local y la comisión de la UEMS, el comité evaluador se puso en contacto con el organizador local para la coordinación del plan de reunión y de trabajo, coordinación con los residentes y con el resto del personal (trabajadores sociales, psicólogos, enfermeros y terapeutas ocupacionales), etc.

\section{Auditoría}

Se realizaron un total de cinco sesiones en dos días, todas ellas en castellano o en inglés con traducción simultánea (realizada por un residente voluntario). Durante la auditoría, el comité evaluador de la UEMS efectuó reuniones y entrevistas con todos los residentes de psiquiatría de dicho hospital, tutores y supervisores de la UD. La primera reunión se llevó a cabo con todos los participantes en el salón de actos, donde se expusieron el programa docente de la UD y el programa de la auditoría. La segunda reunión se realizó entre el residente senior y todos los residentes de psiquiatría, para discutir sobre su programa de formación, sus puntos fuertes y débiles, y para señalar aquellos aspectos que deseaban mejorar. Las conclusiones más relevantes se expusieron durante una tercera reunión entre todos los residentes y el comité evaluador, a la que asistieron tutores, psicólogos, enfermeros, terapeutas ocupacionales y trabajadores sociales, con el fin de debatir otros aspectos positivos y negativos que influían en la formación de los residentes de dicho hospital.

Estas tres reuniones concluyeron con una visita de las infraestructuras destinadas al servicio de psiquiatría, la unidad de psiquiatría y la unidad de urgencias, con el fin de valorar otros aspectos fundamentales inherentes al proceso formativo: medidas de seguridad en la urgencia, dispositivos de guardia, unidades específicas, historias clínicas (redacción, información recogida, prescripción de fármacos comerciales o genéricos...), etc.

Por la tarde, el comité evaluador se reunió en privado para formular conclusiones en base a la información extraída. Se plantearon recomendaciones de mejora a modo de resumen que posteriormente se expusieron ante todo el equipo de psiquiatría de dicho hospital. La quinta reunión se celebró el segundo día: se convocó a todos los participantes al salón de actos para dar un informe escrito, hacer una pequeña presentación oral y expresar su 
opinión sobre la visita. Además, se señaló la necesidad de repetir este mismo estudio pasados cuatro años, para revisar y verificar la consecución de los objetivos acordados. Con esto quedaría cerrado el ciclo de la auditoria y se podría iniciar una nueva.

\section{Resultados}

Los resultados de esta experiencia se pueden resumir desde tres puntos de vista: aspectos positivos, oportunidades de mejora y acciones correctoras.

\section{Aspectos positivos}

Se pueden extraer varios aspectos positivos: se trató de una evaluación global y objetiva, brindó la oportunidad a los residentes a los tutores y supervisores, reforzó la posición del tutor y otros residentes en la negociación, estimuló el compromiso, motivó el cambio y la mejora al ser evaluados y reconocidos externamente por un comité europeo, contribuyó eficazmente a evitar las actividades rutinarias producto de la inercia de las grandes UD, permitió a la especialidad de psiquiatría reconocer el papel del resto de personal psiquiátrico (psicólogos, trabajadores sociales, terapeutas ocupacionales y enfermeros) y fomentó la comunicación e intercambio entre ellos mismos, pudo ofrecer un papel significativo para las asociaciones en la guía y estructuración del proceso de formación, y sirvió como función diplomática que recuerda a los jefes de departamento, supervisores o tutores que ellos tienen la responsabilidad de apoyar la formación docente.

\section{Oportunidades de mejora}

Las principales limitaciones detectadas tuvieron que ver con la supervisión y evaluación de la formación de los residentes, carencias en algunas rotaciones específicas, limitaciones en diversos dispositivos e infraestructura, así como en el control de la práctica clínica diaria. Estas deficiencias fueron similares a las encontradas en otros países [19].

\section{Acciones correctoras}

- Mejora en la evaluación del residente, con pruebas más objetivas e hincapié en el carácter prioritario de la docencia.

- Fomentar que todos los adjuntos, no sólo el tutor, asuman el papel de orientación y supervisión de la formación del residente. Ampliar el tiempo de supervisión al menos en una hora de revisión semanal. Nombrar a un tutor como director de la formación, con un reconocimiento oficial y con la disposición de tiempo necesario para ello.

- Revisión y mejora de los dispositivos indispensables en la práctica clínica diaria y en los servicios de urgencias.

- Reforzar los programas de formación teórica y práctica allí donde fueron más débiles y motivar al cumplimiento.

- Fomentar y animar a los residentes al proceso de asociación que permita la coordinación y participación de ámbito nacional y europeo.

\section{Discusión}

A pesar de los avances en los procesos de auditoria de psiquiatría en España, los sistemas de evaluación de la calidad de formación de los especialistas no reflejan con claridad las deficiencias de la formación de los residentes, futuros especialistas. En ocasiones, estos sistemas de evaluación de calidad se confunden con la calidad investigadora o asistencial. Por otro lado, cuando se ha tratado de conocer la valoración y opinión de tutores y residentes, se han utilizado encuestas que, en general, han tendido a mostrar insatisfacción [20].

De esta manera, y aunque desde 1970 existe un interés creciente por la formación de los especialistas en psiquiatría [21], el cómo se establece y cómo se evalúa esta formación en nuestro país presenta todavía algunas limitaciones, como se puede concluir de la experiencia realizada:

- La evaluación del residente recae fundamentalmente sobre el supervisor de la rotación que el MIR se encuentre realizando en ese momento. Esta estimación resulta muchas veces difícilmente objetiva teniendo en cuenta que ambos, residente y adjunto, adquirirán una relación (después de un período largo de rotación que oscila entre tres y seis meses) que influirá inevitablemente en la subjetividad con la que se valorará la actividad del residente. No se realiza ninguna prueba final que permita valorar, de manera imparcial, el grado de consecución de los objetivos por parte de los residentes, en sus muy diversas competencias.

- Existe un tutor por cada cuatro residentes. Sin embargo, las reuniones con éste terminan siendo escasas por la falta de tiempo y la falta de reconocimiento real de esta labor, lo que invita a la omisión.

- Existe una docencia estipulada y obligatoria semanal, pero en ocasiones no se cumple o resulta insuficiente. 
- Cuando se trata de verificar el grado de satisfacción con respecto al servicio al que se encuentran adscritos (residentes, adjuntos y tutores), se utilizan encuestas anónimas. A pesar de ello no siempre es fácil hacer una crítica objetiva de la unidad, bien por desconocimiento de lo que se hace en otras, bien porque sus opiniones puedan utilizarse negativamente. Algunas encuestas de satisfacción ponen de manifiesto que el programa de especialidad no se llevaba a cabo correctamente [2]. Otra encuesta publicada en 2005 (Asociación de Residentes de Psiquiatría de Cataluña) mostró que la mitad de los residentes en formación estaban poco o nada satisfechos con la tutoría que recibían y un reciente estudio sobre otra encuesta, llevada a cabo por la CN entre los MIR de psiquiatría, mostró un porcentaje sustancial del residentes que no cumplieron de forma adecuada su formación en psicoterapia, metodología de investigación, gerontopsiquiatría, neurología y medicina general [20].

Finalmente, las auditorías que se realizan en España son llevadas a cabo por medio del Ministerio de Sanidad y Consumo a propuesta del comité de docencia de las distintas UD. De esta manera, las auditorías adquieren un carácter más general y aspectos como la profundización en la formación del residente pueden pasarse por alto, 'diluidos' entre todos los aspectos valorados en la auditoría. Así, en ocasiones sucede que las carencias sólo se detectan si hay alguna deficiencia grave. El modelo sugerido por la UEMS trata de que los procesos de auditoría no tiendan a comprobar lo que se subraya en la política normativa de formación, sin reflexionar sobre lo que realmente ocurre (más allá por ejemplo de una docencia estructurada en una UD determinada). De hecho señala que, con frecuencia, se encuentran diferencias entre lo establecido en los programas de formación y en lo que realmente consiste el día a día de un residente [22]. Respecto a los aspectos académicos, varios informes han demostrado diferencias entre las distintas UD tanto en contenido como en los programas de rotación, supervisión y atención a la seguridad del residente [19]. Por este motivo, la UEMS otorga un papel de gran importancia a los profesionales y a los residentes en este proceso de auditoría, invitándolos a adoptar una actitud crítica y constructiva. Especialmente, la trascendencia de la figura del representante de residentes en el proceso de auditoría se ha ido reconociendo con las dificultades encontradas en otros países: los médicos en formación, inseguros ante la autoridad externa, sentían la necesidad de ser leales a sus supervisores y a las instituciones, y temían realizar comentarios que pudieran luego utilizarse en perjuicio de su carrera [12]. La posibilidad de que los residentes puedan reunirse con otro 'igual' (residente), ajeno a su hospital, para trasladar sus inquietudes sobre la formación recibida, favorece la comunicación y localización de los aspectos mejorables de la UD auditada.

Este estudio mediado por la UEMS en el HUPA invita a la reflexión sobre aspectos importantes en el proceso de auditoría, en lo que respecta a la parte de evaluación de la formación de los residentes de psiquiatría, con el objetivo de garantizar que éstos obtengan el máximo nivel en su especialización. Se tratará de fomentar que la profesión psiquiátrica en sí (no sólo el ministerio) asuma la responsabilidad de los niveles de formación y los mantenga en revisión periódica. La incorporación de estos aspectos y la consulta de los estándares utilizados por otros países de la UE facilitarán la aproximación a los estándares de formación de otros países europeos y el movimiento de psiquiatras entre sus fronteras. Ya se han dado dos grandes pasos en esta dirección: de un lado, se ha firmado un acuerdo de colaboración y coordinación en el desarrollo del programa EUMIR entre la SEP y la AEN. Este programa reúne una serie de procedimientos para asegurar la supervisión de la formación de las distintas UD de psiquiatría en España basándose en las recomendaciones de la UEMS [24]. Por otro lado, los residentes han iniciado la creación de una asociación nacional que les permitirá tener una representación y una participación activa en los procesos de formación.

Todos estos procedimientos estarán llenos de marchas y contramarchas. Será necesario repetir el proceso pasados cuatro años para evaluar si se han alcanzado los objetivos acordados. La repetición de esta experiencia en otros centros o la incorporación de los nuevos aspectos en nuestro actual modelo de auditoría serán necesarios para verificar las ventajas de este modelo.

Bibliografía

1. Gómez-Beneyto M, Angosto T. Psychiatric training in Spain. Eur Arch Psychiatry Clin Neurosci 1997; 247: S26-8.

2. Orejas-Pérez O, Martí-Bonany J, Campillo-Sanz T. Programa, organización y estructura de la formación en psiquiatría. SEP / SEPB / AEN. Manual del residente en psiquiatría. Tomo 1. Madrid; 2009. p. 87-92.

3. Comisión Nacional de la Especialidad de Psiquiatría: psiquiatría. Separata de la guía de formación de especialistas. Consejo Nacional de Especialidades Médicas. Ministerio de Sanidad y Consumo. Ministerio de Educación y Cultura 1996; 526: 38.

4. Comisión Nacional de la Especialidad de Psiquiatría: plan de formación de médicos especialistas en psiquiatría. Madrid: Ministerio de Sanidad y Consumo; 1979. 
5. Comisión Nacional de la Especialidad de Psiquiatría: plan de formación de médicos especialistas en psiquiatría. Madrid: Ministerio de Sanidad y Consumo; 1986.

6. Consejo General de Colegios Médicos. Guía de especialidad de psiquiatría. Madrid: CGCM; 1990.

7. Asociación Española de Residentes de Psiquiatría (AERP). 2007. Documentos, estatutos. URL: http://www.psiquiatria. com/documentos/psiq_general_y_otras_areas/otras_areas/ sociedades/aerp_asociacion_espanola_de_residentes_de_ psiquiatria/6588/. [01.01.2012].

8. Plan de calidad del Sistema Nacional de Salud. 2006-2010. Informe Plan de Calidad para el SNS. URL: http://www. msc.es/organizacion/sns/planCalidadSNS/docs/ InformePlanCalidad_ESP.pdf. [01.01.2012].

9. Plan de calidad del Sistema Nacional de Salud. 2009. Cuestionario de psiquiatría. URL: http://www.msc.es/ organizacion/sns/planCalidadSNS/pdf/excelencia/cuestionario/ Psiquiatria.pdf. [01.01.2012].

10. Plan de auditorías docentes. 2006. Ministerio de Sanidad y Consumo. URL: http://www.msps.es/organizacion/sns/ planCalidadSNS/pdf/excelencia/opsc_ac1.pdf. [01.01.2012].

11. Solicitud de acreditación o ampliación docente en psiquiatría. 2007. URL: http://www.san.gva.es/cas/prof/formacion/pdf/ gr46.pdf. [01.01.2012].

12. Warrington JY, Edmonstone G, Strachan J. College approval visits: a perspective on trainee participation. Psychiatrist 1996; 20: 348-50.

13. European Board of Psychiatry. 2003. Accreditation of European training schemes in psychiatry. URL: http://www.uemspsychiatry. org/board/boardReports.htm. [01.01.2012].
14. European Board of Psychiatry. Requirements for the specialist training in psychiatry. Eur Arch Psychiatry Clin Neurosci 1997; 247 (Suppl): 45-7.

15. European Board of Psychiatry. October 2007. Quality assurance in specialist training in psychiatry. URL: http://www. uemspsychiatry.org/common/commonArchivedReports.htm. [01.01.2012].

16. European Federation of Psychiatry Trainees. 2012. URL: http://www.efpt.eu/staticpages/index.php?page= 20080717003224513. [01.01.2012].

17. Prinz R. Erste Deutsche Klinikvisitation durch die UEMS. Young Psychiatrists Newsletter 2005; 76: 371-2.

18. European Board of Psychiatry. October 2002. Training Scheme Assessment Form. URL: http://www.uemspsychiatry.org/ board/forms/schemeAssess.pdf. [01.01.2012].

19. Strachan JG. Training in Europe in perspective. Int Psychiatry 2007; 4: 33.

20. Gómez-Beneyto M, Montilla-García JF, De Castro-Manglano P. La opinión de los residentes de psiquiatría sobre la formación que reciben. Actas Españolas de Psiquiatría 2011; 39: 174-9.

21. López-Ibor JJ, Lenz G., Training and education in psychiatry. Psychol Med 1984; 15: 700-2.

22. European Board of Psychiatry. October 2008. Audit of European Training Schemes in Psychiatry. URL: http:// uemspsychiatry.org/board/reports/TrainingSchemeAudit-2008. pdf. [01.01.2012].

23. Noticias Médicas. La AEN y la SEP firman un convenio para el proyecto EUMIR. Julio 2011. URL: http://www.psiquiatria. com/noticias/psiq_general_y_otras_areas/52684. 